Tohoku J. exp. Med., 1976, 118, 387-392

\title{
An Evaluation of the Function of New Special Equipment for Transrectal Ultrasonotomography
}

\author{
Hiroki Watanabe, * Dairoku Igari, Yoshikatsu Tanahashi, \\ Kazuya Harada and Masahito Saitoh* \\ Department of Urology, Tohoku University School of Medicine, \\ Sendai
}

\begin{abstract}
Watanabe, H., Igari, D., Tanahashi, Y., Harada, K. and Saitoh, M. An Evaluation of the Function of New Special Equipment for Transrectal Ultrasonotomography. Tohoku J. exp. Med., 1976, 118 (4), 387-392_ A new type of equipment for transrectal ultrasonotomography was described in a previous paper. This equipment was evaluated from the data obtained from 205 cases in which examinations were performed. Tomograms were successfully recorded in $94 \%$ of cases. Tomograms sufficiently useful for diagnosis and the measurement of organs were obtained in $89 \%$ of cases for the prostate, $78 \%$ for the urinary bladder, and $80 \%$ for the seminal vesicles. No basic problems in the procedure were found, although hypotension of unknown origin was observed during the examination in a few cases. __ transrectal ultrasonotomography; complication; prostate; urinary bladder; seminal vesicles
\end{abstract}

A clinically useful ultrasonotomogram of intrapelvic organs through the transrectal route was successfully obtained in 1967 (Watanabe et al. 1968; Watanabe et al. 1971) and a new equipment was developed for the procedure in 1973 (Watanabe et al. 1974). Since then, this equipment has been used to examine more than 400 patients. In this report, the function of the equipment will be evaluated on the basis of data obtained from these cases, while clinical evaluations of the procedure have been reported elsewhere (Watanabe et al. 1975; Tanahashi et al. 1975).

\section{Methods and Materials}

The new special equipment for transrectal ultrasonotomography previously described (Watanabe et al. 1974) was employed for the investigation.

During the 19-month period from January, 1973 to July, 1974, examinations were performed in 205 patients of whom 193 were male $(94 \%)$ and 12 were female $(6 \%)$. Diseases of the cases are shown in Table 1. The patients ranged in age from 9 to 87 years.

The tomograms were recorded on $35 \mathrm{~mm}$ black and white film in the usual manner.

\section{RESULTS}

Recording of tomograms

Tomograms were successfully recorded in $193(94 \%)$ of 205 patients. Exposures

Received for publication, December 9, 1975.

* Present address: Department of Urology, Kyoto Prefectural University of Medicine, Kyoto 602 . 
TABLE 1. Number of cases examined

\begin{tabular}{lccc}
\hline \multicolumn{1}{c}{ Disease } & Male & Female & Total \\
\hline & & & $(\%)$ \\
Prostatic hypertrophy & 82 & - & $\mathbf{8 2}(40)$ \\
Prostatic cancer & 33 & - & $\mathbf{3 3}(16)$ \\
Prostatitis or calculi & 24 & - & $\mathbf{2 4}(12)$ \\
Hypoplasia of genital organs & 11 & 0 & $11(5)$ \\
Bladder cancer & 20 & 9 & $\mathbf{2 9}(14)$ \\
Miscellaneous & 12 & 3 & $15(7)$ \\
Normal subject & 11 & 0 & $11(5)$ \\
Total & 193 & 12 & 205 \\
\hline
\end{tabular}

TABLE 2. Problems encountered in the study

\begin{tabular}{lc}
\hline Faulty electrical or mechanical contact of transducer & $\mathbf{1 4}$ times \\
Faulty display circuit & $\mathbf{5}$ \\
Faulty electrical contact of scanner & $\mathbf{5}$ \\
Loosening of scanner handle & 3 \\
Rupture of rubber balloon & 2 \\
Camera-related problem & $\mathbf{1 5}$ \\
\hline
\end{tabular}

between 11 and 66 (average 29) were taken in each case.

We were unable to obtain adequate records in the remaining 12 cases $(6 \%)$. The causes of failure were as follows: malfunction of the camera ( 8 cases), problems with the display circuit (3 cases), and acute pain on insertion of the transducer into the rectum ( 1 case).

Table 2 summarizes the difficulties in evaluation, including both cases in which the tomogram could not be recorded and cases in which a tomogram was finally recorded despite difficulties.

\section{Complications}

Some patients complained of mild discomfort or pain upon insertion of the transducer into the anus. Distinct pain, however, was observed in only 9 patients $(4 \%)$. Pain was so acute in 1 patient that the examination had to be abandoned, though some tomograms had already been recorded.

We previously reported that asthenic patients occasionally showed symptoms such as yawning, nausea, paleness or temporary unconsciousness with hypotension during the course of the transrectal scanning (Igari et al. 1972). These patients recovered a few minutes after removing the transducer. We pointed out also that in the majority of the cases the cause of these symptoms was absorption through the rectal wall of the Lidocain jelly used as a coupling medium (Igari et al. 1972).

During the present evaluation we used a different type of jelly without local anesthetic and far fewer patients complained of these symptoms. Minor hypotensive episodes, however, appeared in 3 patients $(1.5 \%)$. The examination 
procedure was completed in 2 patients without further risk; but in the remaining patient the scanning was interrupted because of the acute symptom mentioned above. Blood pressure in all patients returned to the normal range immediately after removal of the transducer.

\section{Application to special cases}

Although the examination was performed on many patients with hemorrhoids, there were no serious complaints of pain except those mentioned above.

No special disturbances were noted when the examination was done in an above-knee amputee and in a few patients with dysbasia.

\section{Picture quality}

The picture quality of the tomogram was classified into 3 groups:

Group A: Organs were clearly visualized, diseases were well diagnosed and size or weight measurements of organs were possible (Fig. 1).

Group B: Organs were visualized, but picture quality was insufficient to establish a dignosis or record measurements (Fig. 2).

Group $C$ : The visualization of organs was unclear (Fig. 3).

The picture quality in the 193 cases where the tomograms were successfully recorded is shown in Table 3 .

No examination of the prostate and the uterus fell into Group C. In 12 patients $(6 \%)$ pictures of the urinary bladder were judged as Group C. In 8 of

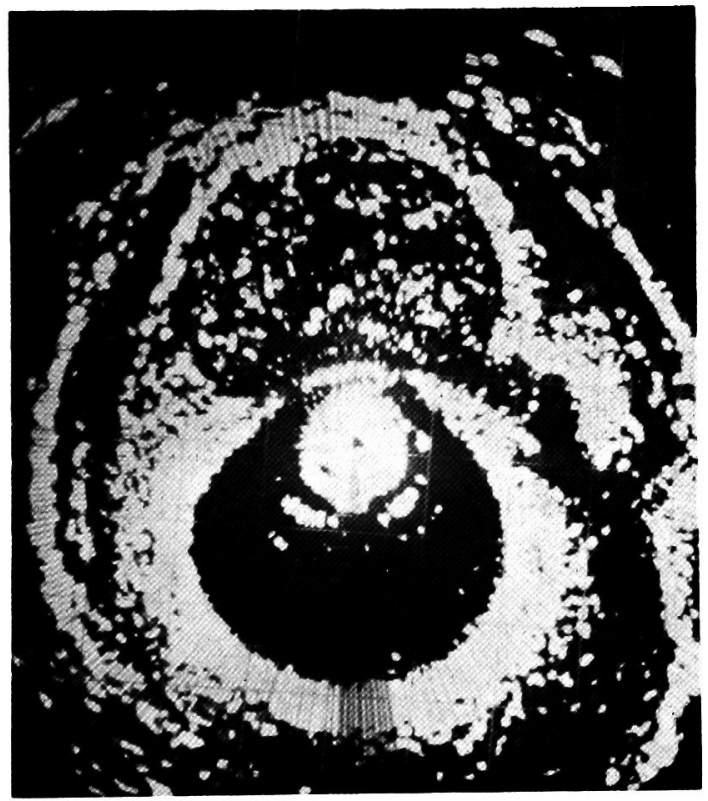

Fig. 1. Tomogram classified into Group A. Prostatic hypertrophy. Horizontal section of the enlarged prostate is clearly visualized. 


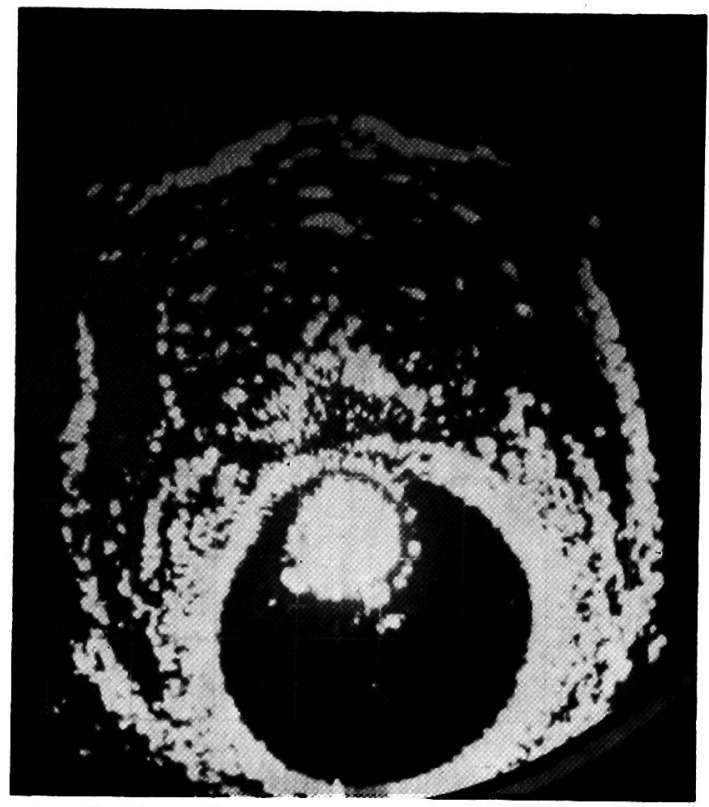

Fig. 2. Tomogram classified into Group B. Prostatic hypertrophy. The enlarged prostate can be recognized but an accurate size measurement is difficult because of an insufficient delineation of the prostatic capsule.

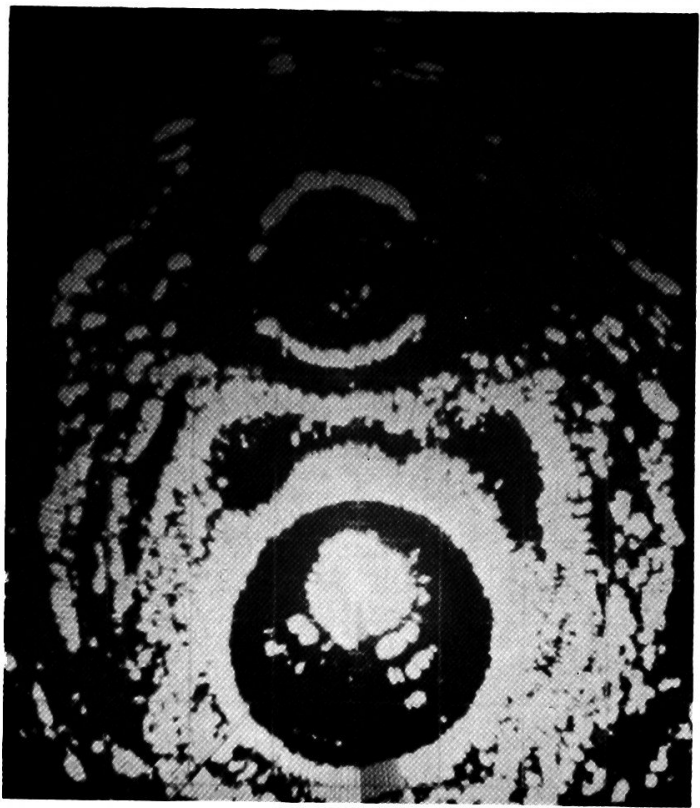

Fig. 3. Tomogram classified into Group C. Prostatic calculi. The contour of the urinary bladder is unclear although an inserted balloon catheter and the seminal vesicles can be distinguished. 
Table 3. Picture quality of tomograms

\begin{tabular}{lcccc}
\hline \multirow{2}{*}{ Organ } & \multicolumn{2}{c}{ Picture quality classification } & Total \\
\cline { 2 - 4 } & $\mathrm{A}$ & $\mathrm{B}$ & $\mathrm{C}$ & \\
\hline & $(\%)$ & $(\%)$ & $(\%)$ & \\
Prostate & $161(89)$ & $20(\mathbf{1 1})$ & 0 & 181 \\
Urinary bladder & $150(\mathbf{7 8})$ & $31(\mathbf{1 6})$ & $12(6)$ & 193 \\
Seminal vesicles & $145(80)$ & $29(\mathbf{1 6})$ & $\mathbf{7}(4)$ & 181 \\
Uterus & $3(25)$ & $9(\mathbf{7 5})$ & 0 & 12 \\
Vagina & $1(8)$ & $8(67)$ & $3(25)$ & 12 \\
\hline
\end{tabular}

A: High quality diagnostic examination. B: Medium quality examination.

C: Poor quality, non-diagnostic examination.

these patients the transducer could not be properly inserted because of a greatly enlarged prostate, in 2 patients the transducer was too close to the bladder and in 2 cases there was trouble in the electrical contact of the scanner.

The tomogram of the seminal vesicles fell into Group C in 7 cases $(4 \%)$ because of echo saturation associated with an oversensitivity of the amplifier in 6 cases and insufficient insertion of the transducer due to pain from hemorrhoids in a remaining case.

\section{Discussion and Conclusion}

This newly developed equipment for transrectal ultrasonotomography was found to be excellent in practice. A clear tomogram sufficient for diagnostic or biometric purpose could be obtained in approximately 90 per cent of all prostate examinations and approximately 80 per cent of examinations of the urinary bladder and seminal vesicles. We feel that these results can be further improved.

Most inadequate examinations were caused by improper operation of the equipment or simple mechanical problems. These problems should be overcome as experience is gained in use of the technique and with further refinement of the equipment.

A few patients developed a hypotensive symptom complex of unknown cause during the examination. Further investigations are necessary to delineate the pathogenesis of these symptoms.

\section{Acknowledgment}

This work was supported by grant for cancer research from the Welfare Ministry of Japan.

\section{References}

1) Igari, D., Watanabe, H., Shima, M., Tanahashi, Y. \& Harada, K. (1972) Ultrasonotomography of the prostate (7th report). Side effect of Lidocain-jelly absorbed through rectal wall. Proc. Jap. Soc. Ultrasonics Med. (Jap.), 22, 95-96.

2) Tanahashi, Y., Watanabe, H., Igari, D., Harada, K. \& Saitoh, M. (1975) Volume estimation of the seminal vesicles by means of transrectal ultrasonotomography. Brit. J. Urol., 47, 695-702. 
3) Watanabe, H., Kato, H., Kato, T., Morita, M., Tanaka, M. \& Terasawa, Y. (1968) Diagnostic application of ultrasonotomography for the prostate. Jap. J. Urol. (Jap.), 59, 273-279.

4) Watanabe, H., Kaiho, H., Tanaka, M. \& Terasawa, Y. (1971) Diagnostic application of ultrasonotomography to the prostate. Invest. Urol., 8, 548-559.

5) Watanabe, H., Igari, D., Tanahashi, Y., Harada, K. \& Saitoh, M. (1974) Development and application of new equipment for transrectal ultrasonography. J. clin. Ultrasound, 2, 91-98.

6) Watanabe, H., Igari, D., Tanahashi, Y., Harada, K. \& Saitoh, M. (1975) Transrectal ultrasonotomography of the prostate. J. Urol., 114, 734-739. 